\title{
RESEARCH
}

Open Access

\section{The impact of transcranial magnetic stimulation on diagnostic confidence in patients with Alzheimer disease}

Alberto Benussi ${ }^{1 \dagger}$, Antonella Alberici ${ }^{1 \dagger}$, Clarissa Ferrari ${ }^{2+}$, Valentina Cantoni ${ }^{1,3}$, Valentina Dell'Era', Rosanna Turrone ${ }^{1}$, Maria Sofia Cotelli ${ }^{4}$, Giuliano Binetti ${ }^{2}$, Barbara Paghera ${ }^{5}$, Giacomo Koch ${ }^{6,7}$, Alessandro Padovani ${ }^{1}$ and Barbara Borroni ${ }^{1 *}$ D

\begin{abstract}
Background: Cholinergic dysfunction is a key abnormality in Alzheimer disease (AD) that can be detected in vivo with transcranial magnetic stimulation (TMS) protocols. Although TMS has clearly demonstrated analytical validity, its clinical utility is still debated. In the present study, we evaluated the incremental diagnostic value, expressed in terms of diagnostic confidence of Alzheimer disease (DCAD; range 0-100), of TMS measures in addition to the routine clinical diagnostic assessment in patients evaluated for cognitive impairment as compared with validated biomarkers of amyloidosis.
\end{abstract}

Methods: One hundred twenty patients with dementia were included and scored in terms of DCAD in a three-step assessment based on (1) demographic, clinical, and neuropsychological evaluations (clinical work-up); (2) clinical work-up plus amyloid markers (cerebrospinal fluid or amyloid positron emission tomographic imaging); and (3) clinical work-up plus TMS intracortical connectivity measures. Two blinded neurologists were asked to review the diagnosis and diagnostic confidence at each step.

Results: TMS measures increased the discrimination of DCAD in two clusters (AD-like vs FTD-like) when added to the clinical and neuropsychological evaluations with levels comparable to established biomarkers of brain amyloidosis (cluster distance of 55.1 for clinical work-up alone, 76.0 for clinical work-up plus amyloid markers, 80.0 for clinical work-up plus TMS). Classification accuracy for the "gold standard" diagnosis (dichotomous - AD vs FTD variable) evaluated in the three-step assessment, expressed as AUC, increased from 0.82 (clinical work-up alone) to 0.98 (clinical work-up plus TMS) and to 0.99 (clinical work-up plus amyloidosis markers).

Conclusions: TMS in addition to routine assessment in patients with dementia has a significant effect on diagnosis and diagnostic confidence that is comparable to well-established amyloidosis biomarkers.

Keywords: Alzheimer disease, Frontotemporal dementia, Transcranial magnetic stimulation, PET amyloid, Diagnosis, Confidence

\footnotetext{
* Correspondence: bborroni@inwind.it

${ }^{\dagger}$ Alberto Benussi, Antonella Alberici and Clarissa Ferrari contributed equally

to this work.

${ }^{1}$ Neurology Unit, Department of Clinical and Experimental Sciences,

University of Brescia, Brescia, Italy

Full list of author information is available at the end of the article
}

(c) The Author(s). 2018 Open Access This article is distributed under the terms of the Creative Commons Attribution 4.0 International License (http://creativecommons.org/licenses/by/4.0/), which permits unrestricted use, distribution, and reproduction in any medium, provided you give appropriate credit to the original author(s) and the source, provide a link to the Creative Commons license, and indicate if changes were made. The Creative Commons Public Domain Dedication waiver (http://creativecommons.org/publicdomain/zero/1.0/) applies to the data made available in this article, unless otherwise stated. 


\section{Background}

The clinical diagnosis of neurodegenerative disorders is based on an extensive evaluation of cognitive and behavioral performance, along with functional status, which provides a variable grade of accuracy, with a definite diagnosis reached only at autopsy $[1,2]$. Many technological advancements have been implemented to serve as surrogates for specific neuropathological hallmarks and to improve the diagnostic work-up of dementia [3]. Over the past decade, many steps have been taken to increase the accuracy of Alzheimer disease (AD) diagnosis, and recent criteria state that positivity of one or more biomarkers of brain amyloidosis or neuronal injury is associated with a high likelihood of $\operatorname{AD}[4,5]$. Furthermore, important innovations in ongoing clinical trials in AD now include the use of preclinical/prodromal biomarkers, considering the increasing evidence that disease-modifying treatments must be administered early in the disease course $[6,7]$. As a result, the development of diagnostic tools capable of accurately discriminating AD from frontotemporal dementia (FTD) at early disease stages has become a crucial target [4].

Decreased levels of amyloid- $\beta$ 1-42 $\left(\mathrm{A} \beta_{1-42}\right)$ in the cerebrospinal fluid (CSF) and/or increased binding of amyloid ligands visualized by positron emission tomography (PET) are the most established and validated amyloid markers [8-11], being helpful in increasing the diagnostic confidence of Alzheimer disease (DCAD) among clinicians $[12,13]$. However, despite the usefulness of these markers, a number of drawbacks, such as invasive procedures (i.e., CSF collection), expensiveness (i.e., PET amyloid), or availability restricted to tertiary dementia centers, may limit their use. Concomitantly, there is a growing demand to identify inexpensive, easy-to-perform, noninvasive, and safe biomarkers to be used extensively on clinical grounds [14].

In this context, our group has recently developed an index using transcranial magnetic stimulation (TMS) intracortical connectivity measures, yielding a diagnostic accuracy of $90 \%$ in identifying AD with high accuracy even in the early phases of disease $[15,16]$. Short-latency afferent inhibition (SAI), assessing the function of cholinergic circuits indirectly, has been found to be impaired in patients with AD [15-17]; conversely, short-interval intracortical inhibition (SICI) and intracortical facilitation (ICF), markers of $\gamma$-aminobutyric acid type $\mathrm{A}\left(\mathrm{GABA}_{\mathrm{A}}\right)$ ergic and glutamatergic neurotransmission, respectively, have been found to be impaired in patients with FTD $[17,18]$. These findings stemmed from the evidence that $\mathrm{AD}$ is defined by both amyloid deposits and a well-established cholinergic deficit [19-22], whereas in FTD, abnormalities in glutamatergic and GABAergic neurotransmission have been reported [23-26].
The assessment of TMS intracortical connectivity holds promise to be a useful tool in the differential diagnosis of neurodegenerative diseases, being free from strict exclusion criteria, not time-consuming, and inexpensive [27]. However, its clinical value needs to be further demonstrated, also taking into consideration that both conditions may show several overlapping features, such as amyloid positivity in FTD [28], cholinergic deficits in FTD [29], or glutamatergic overexpression in $\mathrm{AD}$ [30]. Indeed, several studies now suggest that some cases of AD and FTD are linked in a genetic spectrum of degenerative brain disorders in which tau appears to be the central player [31]. Moreover, this is especially important in patients with late-onset disease, in whom differential diagnosis becomes more challenging owing to overlapping symptoms and mixed neuropathology.

All the above observations defined the objective of this work, aimed at evaluating the clinical utility of TMS compared with amyloid markers in DCAD. To this end, the impact of TMS intracortical connectivity measures was compared with that of amyloid markers when both were added to routine clinical assessment. A validation of DCAD, in terms of prediction performance of a "gold standard" diagnosis, concluded the work.

\section{Methods \\ Approval}

Full written informed consent was obtained from all participants according to the Declaration of Helsinki. The study protocol was approved by the local ethics committee (NP1965; approved 19 May 2015).

\section{Participants and study design}

Patients with either probable AD [5] or FTD [32, 33] were consecutively recruited from the Neurology Unit, Department of Clinical and Experimental Sciences, University of Brescia, Italy.

Probable AD was defined as the presence of cognitive or neuropsychiatric symptoms with an insidious onset that interfered with the ability to function at work or at usual activities, involving a minimum of two cognitive domains, with evidence of the $\mathrm{AD}$ pathophysiological process [5]. Patients with FTD were diagnosed as behavioral variant FTD (bvFTD) [33] or semantic variant FTD (svPPA) and agrammatic variant of primary progressive aphasia (avPPA), according to current clinical criteria [32]. These criteria have shown good correlations in clinicopathological studies [34, 35].

Demographic characteristics, family history, and clinical features were carefully recorded. During the first visit, dementia experts (MSC, AP, and $\mathrm{BB}$ ) performed a neurological, cognitive, and behavioral examination and did a preliminary assessment of eligibility. All patients considered in the present study underwent a standardized 
neuropsychological evaluation, brain magnetic resonance imaging (MRI), at least one biomarker of brain amyloidosis (i.e., CSF $A \beta_{1-42}$ dosage and/or amyloid PET scan), and TMS intracortical connectivity measures, as described below.

Patients with a history of epilepsy or with electronic implants were excluded from the study.

None of the patients were treated with drugs that could have altered the cerebral cortex excitability in the previous 3 months, such as benzodiazepines, acetylcholinesterase inhibitors, neuroleptics, or antidepressants. If patients were already on central nervous system active medications, they were asked to gradually decrease their dosage until suspension, only if this was not accompanied by a relevant deterioration in cognitive, behavioral, or clinical symptoms for which the therapy was restarted, thus excluding the patient from entering the study.

Patients' data were then anonymized, and the following information was presented to two experienced neurologists ( $\mathrm{AB}$ and $\mathrm{AA})$ in three separate sections in which they were made aware of (1) demographic characteristics, family history, clinical and neuropsychological assessment, and structural imaging data (henceforth defined as "clinical work-up"); (2) clinical work-up plus amyloid markers; and (3) clinical work-up plus TMS intracortical connectivity measures.

On the basis of the data obtained in section (1), the two experienced neurologists formulated their etiological diagnosis (AD vs FTD) and rated their confidence that cognitive impairment was due to $\mathrm{AD}$ on a structured scale ranging from $0 \%$ to $100 \%$ (DCAD, $0-100 \%$ ). Thus, DCAD $>50 \%$ supported an AD diagnosis, whereas DCAD $<50 \%$ supported an FTD diagnosis. In cases in which the same diagnosis was reached by both raters, mean DCAD was considered. In the cases in which there was disagreement (one neurologist suggested $A D$ and the other FTD diagnosis), a second round of joint evaluation was carried out, and a shared diagnosis and DCAD agreement was reached.

The same protocol was adopted for section (2) and section (3), in which the two neurologists were asked to revise patients' diagnosis and DCAD after disclosure of combined clinical work-up along with either amyloid markers (section 2) or TMS intracortical connectivity measures (section 3). Thus, any change in diagnosis or DCAD in the subsequent sections could only be attributed to the knowledge of such results.

In each section, patients were presented in a random order, and for each diagnostic disagreement, a second round of joint evaluation was performed, as for section (1). At the end, a shared diagnosis and mean DCAD between the two raters was provided for each patient regarding clinical work-up, clinical work-up plus amyloid markers, and clinical work-up plus TMS measures (see
Fig. 1 for study design). Moreover, a "gold standard" diagnosis (i.e., AD or FTD) was provided by the dementia experts (MSC, AP, and $\mathrm{BB}$ ), who had the patients in charge and who had complete access to all the available information, such as the clinical work-up, amyloid markers, and TMS.

\section{Clinical work-up}

The set of mandatory information for each recruited subject, which was presented to the two neurologists during the clinical work-up evaluation, included demographic characteristics (age, sex, family history, past medical history, and comorbidities), conventional structural brain imaging findings, and the results of the neuropsychological assessment, including global cognitive function, long-term memory, executive function, and language and visual spatial abilities. In particular, for all patients, at least the following tests were available: Mini Mental State Examination (MMSE) [36], Clinical Dementia Rating [37], short story [38], copy and recall of the Rey-Osterrieth complex figure test [39], Trail Making Test part A and part B [40], semantic and phonemic fluency [41], and clock-drawing test. Moreover, basic [42] and instrumental activities of daily living [43] and the Neuropsychiatric Inventory [44] were reported. All the above data were provided to the two raters in sections (1), (2), and (3).

\section{Amyloid markers}

In this group, diagnostic markers of amyloidosis, including CSF $A \beta_{1-42}$ analysis or amyloid PET imaging, were assessed. Lumbar puncture was carried out in the outpatient clinic according to standard procedures, and CSF analysis was performed using an enzyme-linked immunosorbent assay (INNOTEST; Innogenetics, Ghent, Belgium) [45]. Accordingly, a CSF AD-like profile was defined as CSF $\mathrm{A} \beta_{1-42} \leq 650 \mathrm{ng} / \mathrm{L}$ (along with CSF Tau $\geq 400 \mathrm{ng} / \mathrm{L}$ ). Amyloid PET imaging was acquired using $370 \mathrm{MBq}$ $(10 \mathrm{mCi})$ of ${ }^{18} \mathrm{~F}$-florbetapir, and visual readings were performed by a nuclear medicine physician who was blinded to the patients' diagnoses, following the procedures provided by the ligand manufacturer, as previously reported [12]. CSF $A \beta_{1-42}$ dosage (along with CSF tau) and/or amyloid PET result (amyloid PET "positive" vs "negative") were provided to the two raters in section (ii).

\section{Transcranial magnetic stimulation intracortical connectivity measures}

TMS protocols were carried out as previously published [46]. TMS was performed with a figure-of-eight coil (each loop diameter $70 \mathrm{~mm}$ ) connected to a Magstim Bistim $^{2}$ system (Magstim Company, Oxford, UK). Motor evoked potentials (MEPs) were recorded from the right first dorsal interosseous muscle (FDI) through surface $\mathrm{Ag} / \mathrm{AgCl}$ electrodes placed in a belly-tendon montage 


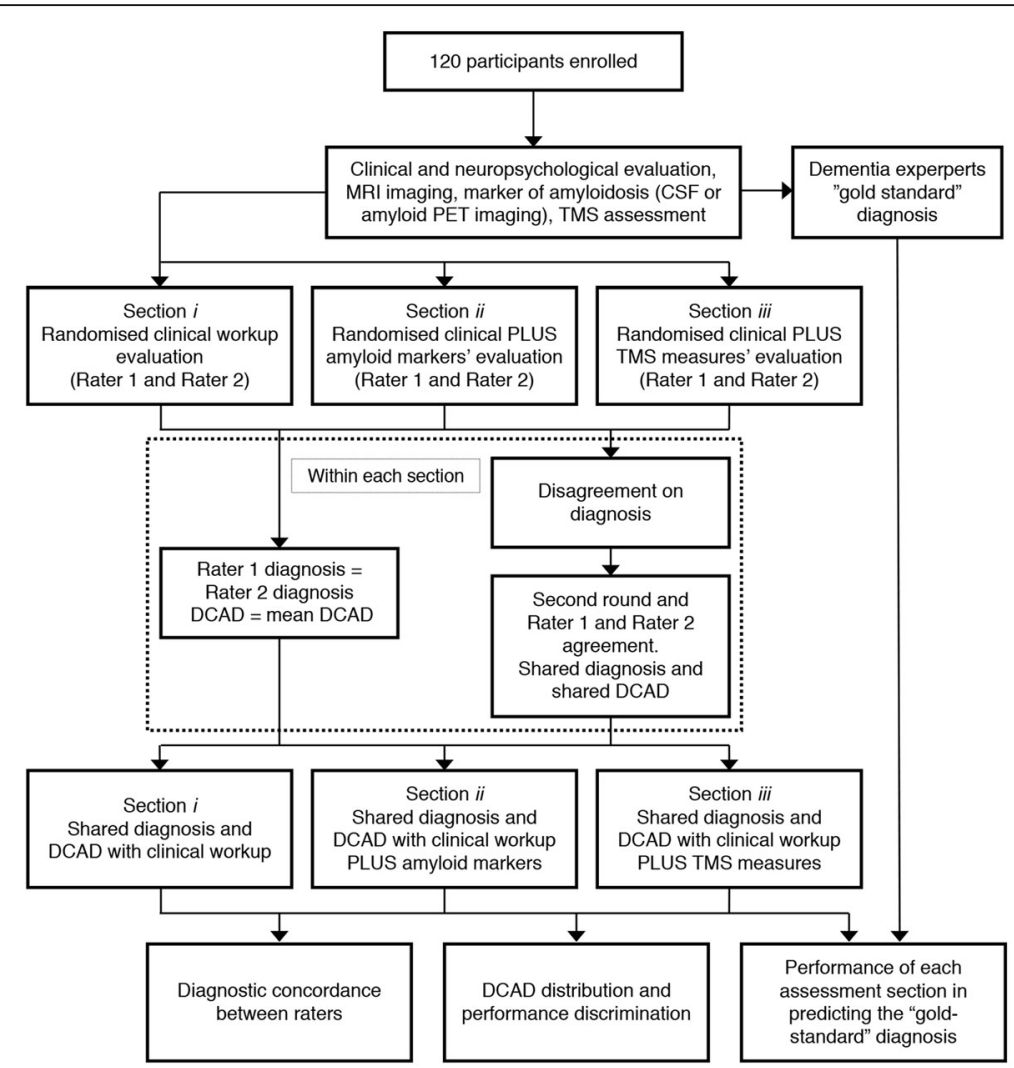

Fig. 1 Study design. CSF Cerebrospinal fluid, DCAD Diagnostic confidence of Alzheimer disease, MRI Magnetic resonance imaging, PET Positron emission tomography, TMS Transcranial magnetic stimulation

and acquired using a Biopac MP-150 electromyograph (BIOPAC Systems Inc., Santa Barbara, CA, USA). The TMS coil was held tangentially over the scalp region corresponding to the primary hand motor area contralateral to the target muscle, with the coil handle pointed 45 degrees posteriorly and laterally to the sagittal plane. The motor hot spot was defined as the location where TMS consistently produced the largest MEP size at $120 \%$ of the resting motor threshold (rMT) in the target muscle.

rMT was defined as the minimal stimulus intensity needed to produce MEPs with an amplitude of at least $50 \mu \mathrm{V}$ in five of ten consecutive trials during complete muscle relaxation, which was controlled by visually checking the absence of electromyographic (EMG) activity at high-gain amplification [47].

We considered SICI and ICF, which predominantly reflect $\mathrm{GABA}_{\mathrm{A}}$ ergic and glutamatergic neurotransmission, respectively $[48,49]$, and SAI, which primarily reflects cholinergic transmission, using a previously described technique [50]. SICI and ICF were studied at rest via a paired-pulse paradigm, delivered in a conditioning test design with the conditioning stimulus (CS) set at an intensity of $70 \%$ of the rMT, whereas the test stimulus
(TS) was adjusted to evoke an MEP of approximately $1 \mathrm{mV}$ peak to peak in the relaxed FDI. Different interstimulus intervals (ISIs) between the CS and TS were employed to investigate preferentially both SICI $(1,2,3$, and $5 \mathrm{~ms})$ and ICF $(7,10$, and $15 \mathrm{~ms})[48,49]$.

SAI, which primarily reflects cholinergic transmission, was studied using a previously described technique [50]. CSs were single pulses $(200 \mu \mathrm{s})$ of electrical stimulation applied through bipolar electrodes to the right median nerve at the wrist (cathode proximal). The intensity of the CS was set at just over motor threshold for evoking a visible twitch of the thenar muscles, whereas the TS was adjusted to evoke an MEP of approximately $1 \mathrm{mV}$ peak to peak. The CS to the peripheral nerve preceded the TS by different ISIs $(-4,0,+4,+8 \mathrm{~ms}$, determined relative to the latency of the N20 component of the somatosensory evoked potential).

Ten stimuli were delivered for each ISI for all stimulation paradigms, and fourteen control MEPs in response to the TS alone, were recorded for each paradigm in all participants in a pseudorandomized sequence. The amplitude of the conditioning MEPs was expressed as a ratio of the mean unconditioned response. The intertrial interval was set at $5 \mathrm{~s}( \pm 10 \%)$. 
SICI-ICF and SAI protocols were performed in a randomized order. Throughout the experiment, complete muscle relaxation was monitored by audiovisual feedback when appropriate. If the quality of study data was degraded by patient movement, the protocol was recommenced, and the initial data were discarded. Trials were discarded if EMG activity exceeded $100 \mu \mathrm{V}$ in the $250 \mathrm{~ms}$ prior to TMS stimulus delivery. All patients were able to understand instructions and obtain full muscle relaxation.

The SICI-ICF/SAI ratio was defined as average SICI $(1,2,3 \mathrm{~ms})$ /average ICF $(7,10,15 \mathrm{~ms})$ /average SAI $(0,+$ $4 \mathrm{~ms})$. The SICI-ICF/SAI ratio was provided to the two raters, and they considered the previous published cutoff value of 0.98 [15] in section (iii). The operators who performed TMS (VC and VD) were blinded to the subjects' amyloid marker status and clinical/neuropsychological evaluation.

\section{Statistical analysis}

Sociodemographic characteristics of the patients as well as descriptive features of the DCAD were provided through mean, SD, and median values. To assess the correlation and agreement of DCAD between the two raters, and between each rater and the shared DCAD, nonparametric Spearman's correlation, and intraclass correlation coefficient (ICC; single measures) were used.

A data-driven model-based classification method, namely a Gaussian mixture model $[51,52]$, was applied to evaluate the performance of the three different assessments (clinical work-up, clinical work-up plus amyloid markers, and clinical work-up plus TMS) in discriminating between $\mathrm{AD}$ and FTD. A mixture model procedure allows one to obtain a probabilistic clustering that quantifies the uncertainty of the data belonging to components (clusters) of the mixture. The estimation procedure was carried out using an expectation-maximization algorithm [53], and the number of clusters was estimated on the basis of the Bayesian information criterion (BIC) index for goodness of model fit (the lower the BIC, the better the model fits) [54]. Once clusters for each assessment DCAD distribution were detected through mixture models, two different indices were carried out to evaluate the discriminative performance in each assessment section: the cluster centroid distance (i.e., distance, in terms of DCAD, between the two cluster middles) and the DCAD mean with $95 \% \mathrm{CI}$ of the mixture clusters. In particular, for the cluster means (i.e., the means of the Gaussian components of each mixture model), statistical difference was evaluated through $t$ tests.

Finally, the association of DCAD of each of the three sections (independent variables) with the "gold standard" diagnosis (dichotomous dependent variable) was evaluated through logistic regression models. Performance of each assessment section in predicting the "gold standard" diagnosis was evaluated through ROC curves and the corresponding AUC values applied on predictive probability scores obtained by the logistic models. High values of AUC (>0.8) indicate good performance of independent variables in predicting the diagnosis and thus in classifying $\mathrm{AD}$ vs FTD. Comparison of AUC was performed using the DeLong test.

Statistical significance was assumed at $p<0.05$. Data analyses were carried out using the 'mclust' and 'InformationValue' packages of $\mathrm{R}$ statistical software (http:// www.r-project.org).

\section{Results}

\section{Participants and TMS measures}

Among 120 patients, 57 (47.31\%) were female, the mean age was $67.5 \pm 8.36$ years, the mean age at onset was $64.1 \pm$ 7.90 years, and the mean MMSE score was $23.5 \pm 5.93$. According to the "gold-standard" diagnosis, 63 patients were classified as $\mathrm{AD}$ (mean age, $70.3 \pm 7.2$; female sex, $52.4 \%$; MMSE score, $22.8 \pm 5.6$ ) and 57 as FTD (mean age, 64.4 \pm 8.5; female sex, 42.1\%; MMSE score, $24.2 \pm 6.3$ ). Demographic, clinical, and neurophysiological scores for each group of patients are reported in Table 1. In the FTD group, 42 patients were classified as bvFTD, 8 as avPPA, and 7 as svPPA. No significant differences in average SICI $(p=0.161)$, ICF $(p=0.936)$, or SAI $(p=0.678)$ were observed between FTD subgroups. All procedures, including TMS sessions, were generally well tolerated with no adverse events reported in the whole cohort of patients.

\section{Diagnostic concordance between raters}

A high correlation between the two raters (AA's diagnosis vs AB's diagnosis) and between each rater and shared diagnosis (AA's diagnosis vs shared diagnosis and AB's diagnosis vs shared diagnosis) was reported in all three sections (Spearman's correlation coefficient ranges, 0.65 to 0.86 in clinical work-up section; 0.82 to 0.91 in clinical work-up plus amyloid markers; 0.79 to 0.85 in clinical work-up plus TMS). Similarly, high values of ICC (AB's diagnosis, AA's diagnosis, and shared diagnosis) were found for clinical work-up (ICC, 0.74), and these increased when clinical work-up plus amyloid markers (ICC, 0.88) or clinical work-up plus TMS (ICC, 0.94) was considered. The high values of correlation and agreement legitimize the use, hereafter, of the shared diagnosis (i.e., the mean DCAD between the two raters) as the main outcome variable for assessing DCAD of each assessment section.

DCAD distribution and performance discrimination of the three assessment sections

A bimodal distribution was found for DCAD in all three section assessments. The Gaussian mixture model approach 
Table 1 Demographic, clinical, and neurophysiological characteristics of included patients

\begin{tabular}{|c|c|c|c|}
\hline & $A D$ & FTD & $p$ Value* \\
\hline Patients (number) & 63 & 57 & - \\
\hline Age, years & $70.3 \pm 7.2$ & $64.4 \pm 8.5$ & $p<0.001$ \\
\hline Age at onset (years) & $66.9 \pm 6.9$ & $60.9 \pm 7.8$ & $p<0.001$ \\
\hline Sex (\% female) & 52.4 & 42.1 & n.s. \\
\hline Education (years) & $10.1 \pm 4.7$ & $10.2 \pm 4.5$ & n.s. \\
\hline MMSE & $22.8 \pm 5.6$ & $24.2 \pm 6.3$ & n.s. \\
\hline CDR & $0.9 \pm 0.5$ & $0.9 \pm 0.6$ & n.s. \\
\hline NPI & $10.9 \pm 8.4$ & $16.8 \pm 10.6$ & $p=0.002$ \\
\hline Short story & $3.9 \pm 3.2$ & $8.3 \pm 11.0$ & $p=0.009$ \\
\hline Rey figure copy & $19.3 \pm 11.7$ & $24.8 \pm 9.4$ & $p=0.022$ \\
\hline Rey figure recall & $4.0 \pm 5.2$ & $8.2 \pm 6.4$ & $p=0.001$ \\
\hline TMT-A (s) & $179.2 \pm 177.5$ & $112.8 \pm 136.8$ & $p=0.053$ \\
\hline TMT-B (s) & $437.8 \pm 152.7$ & $345.3 \pm 175.4$ & $p=0.008$ \\
\hline Phonemic fluency & $21.6 \pm 9.6$ & $16.6 \pm 10.9$ & $p=0.023$ \\
\hline Semantic fluency & $20.3 \pm 12.3$ & $32.5 \pm 70.2$ & n.s. \\
\hline Clock-drawing test & $5.5 \pm 2.8$ & $6.7 \pm 2.8$ & $p=0.028$ \\
\hline BADL lost & $0.3 \pm 0.9$ & $0.6 \pm 1.2$ & n.s. \\
\hline IADL lost & $1.8 \pm 2.2$ & $1.8 \pm 2.3$ & n.s. \\
\hline Amyloid PET, number positive & $38 / 38$ & $1 / 7$ & $p<0.001$ \\
\hline \multicolumn{4}{|l|}{ Cerebrospinal fluid } \\
\hline $\mathrm{t}-\mathrm{Tau}$ & $788.2 \pm 414.5$ & $317.0 \pm 177.6$ & $p<0.001$ \\
\hline $\mathrm{p}-\mathrm{Tau}_{181}$ & $129.4 \pm 213.5$ & $44.2 \pm 24.1$ & $p=0.027$ \\
\hline$A \beta_{1-42}$ & $520.3 \pm 109.6$ & $900.6 \pm 291.3$ & $p<0.001$ \\
\hline \multicolumn{4}{|l|}{ TMS measures } \\
\hline $\mathrm{SICl}$ & $0.29 \pm 0.11$ & $0.67 \pm 0.26$ & $p<0.001$ \\
\hline ICF & $1.41 \pm 0.20$ & $0.87 \pm 0.22$ & $p<0.001$ \\
\hline SAl & $0.84 \pm 0.10$ & $0.52 \pm 0.12$ & $p<0.001$ \\
\hline SICI-ICF/SAI ratio & $0.26 \pm 0.11$ & $1.67 \pm 0.91$ & $p<0.001$ \\
\hline
\end{tabular}

Abbreviations: $A \beta_{1-42}$ Amyloid- $\beta$ 1-42, AD Alzheimer disease, $B A D L$ Basic activities of daily living, $C D R$ Clinical Dementia Rating, FTD Frontotemporal dementia, IADL Instrumental activities of daily living, ICF Average intracortical facilitation (7, 10, 15 ms), IS/ Interstimulus interval, MMSE: Mini Mental State Examination, n.s. Not significant, NPI Neuropsychiatric Inventory, PET Positron emission tomography, $p$-Tau ${ }_{181}$ Phosphorylated tau 181 , SAl Average shortlatency afferent inhibition $(0,+4 \mathrm{~ms})$, SICl Average short-interval intracortical inhibition (1, 2, 3 ms), SICI-ICF/SAI Average SICI at ISI 1, 2, $3 \mathrm{~ms} /$ average ICF at ISI 7, 10, $15 \mathrm{~ms}$ /average SAI at ISI 0, +4 ms, TMS Transcranial magnetic stimulation, TMT-A Trail Making Test part A, TMT-B Trail Making Test part B, $t$-tau Total tau

Demographic and clinical characteristics and neurophysiological parameters are expressed as mean \pm SD (unless otherwise specified)

* $p$ Values for independent samples $t$ test or Fisher's exact test, as appropriate

identified two clusters (lower BIC indices were obtained by mixture models with two-component clusters) in each of the three DCAD distributions: the FTD-like cluster (characterized by lower DCAD values) and the AD-like cluster (defined by higher DCAD values).

In Fig. 2, mixture model fitting estimations (Fig. 2a) and corresponding cluster identification (Fig. 2b) are reported. The greater the distance between the two peaks, the higher the discrimination performance between the two diagnoses.

In Table 2, group centroids' distance of the two DCAD clusters as well as their means and 95\% CIs are reported. Although cluster mean differences were statistically different for all the three assessment sections $\left(p<1.0 \times 10^{-}\right.$ ${ }^{5}$ for all), the lower separation between the two peaks, and thus the lower discrimination performance, was found with clinical work-up (cluster distance equal to 55.1), whereas the separation increased when clinical work-up plus amyloid markers was considered (cluster distance of 76.0) and further increased when clinical work-up plus TMS was analyzed (cluster distance equal to 80.0 ).

\section{Performance of each assessment section in predicting the "gold standard" diagnosis}

Logistic regression models revealed a high statistically significant association between "gold standard" diagnosis and all the three assessments ( $p<0.046$ for all). In detail, an enhancement of one unit in DCAD value corresponds to an increased probability to reach a diagnosis of $\mathrm{AD}$ of $5 \%, 11 \%$, and $32 \%$ (ORs equal to $1.05,1.11$, and 1.32, respectively), for clinical work-up, clinical work-up plus amyloid markers, and clinical work-up plus TMS assessment, respectively.

Considering the performance in predicting the "gold standard" diagnosis, although all three assessments reached high values of specificity and sensitivity in classifying AD vs FTD correctly (AUC $>0.8$ for all), the best performance was obtained by clinical work-up plus amyloid markers and by clinical work-up plus TMS measures (see Table 3). AUCs of clinical work-up plus amyloid markers and of clinical work-up plus TMS were statistically different from the AUC of clinical work-up (De Long test $p=2.6 \times 10^{-5}$ and $p=4.1 \times 10^{-5}$, respectively), and, interestingly, these were not different from each other $(p=0.619)$ (see Additional file 1: Figure S1).

\section{Discussion}

It has been widely demonstrated that the DCAD can be improved by the use of biological markers [12]. A number of biomarkers of functional impairment, neuronal loss, and protein deposition that can be assessed by neuroimaging (i.e., MRI and PET) or CSF analysis have been validated to diagnose $\mathrm{AD}$ in research studies and specialist clinical settings so far [3]. Some of these biomarkers have shown very high diagnostic accuracy in discriminating AD from FTD. Indeed, previous studies have shown that fluorodeoxyglucose (FDG)-PET has a diagnostic accuracy of up to $87 \%$ in differentiating $\mathrm{AD}$ from FTD, amyloid PET imaging has a diagnostic accuracy of $97 \%$ [55], and CSF analysis has a diagnostic accuracy of up to 


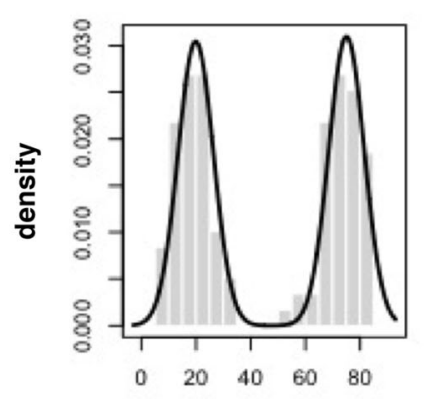

DCAD with clinical work-up

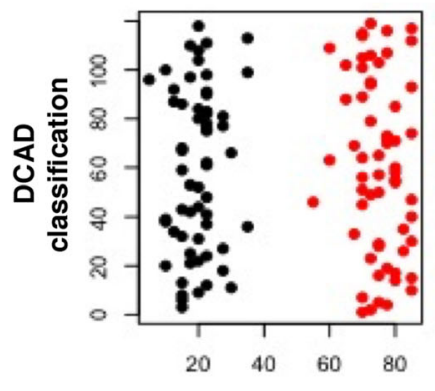

DCAD with clinical work-up

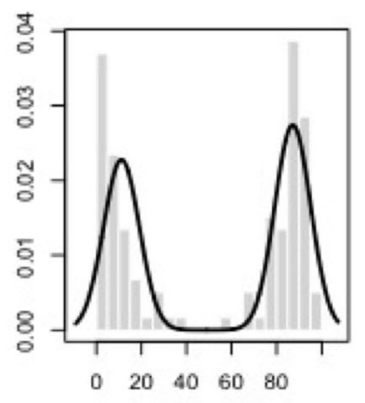

DCAD with clinical PLUS amyloid markers

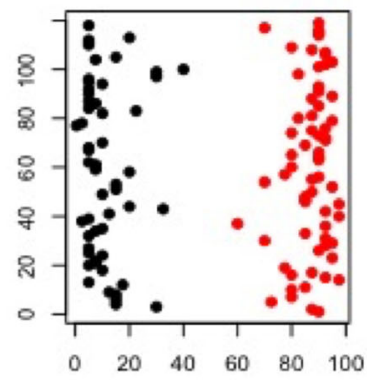

DCAD with clinical PLUS amyloid markers

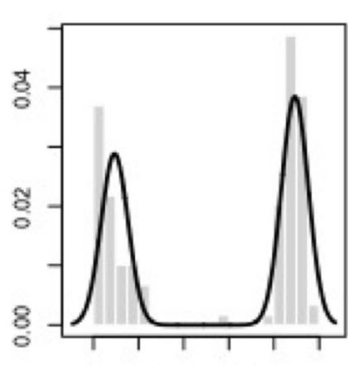

$\begin{array}{lllll}0 & 20 & 40 & 60 & 80\end{array}$

DCAD with clinical PLUS TMS markers

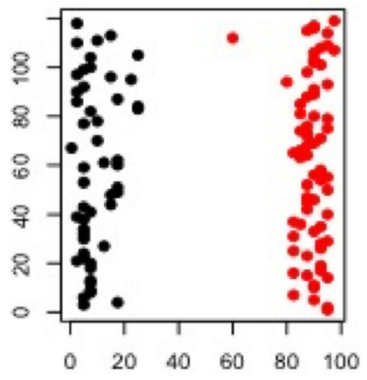

DCAD with clinical PLUS TMS markers

Fig. 2 Mixture model estimation (upper panels) and classification (lower panels; red dots, Alzheimer disease group; black dots, frontotemporal dementia group). DCAD Diagnostic confidence of Alzheimer disease, TMS Transcranial magnetic stimulation

95\% [56]. Each biomarker has been proven reliable and accurate, even though a number of drawbacks might limit its use in clinical settings. FDG-PET, which provides important information on the topographical distribution of the ongoing neurodegenerative process and thus limited in the differential diagnosis of focal variants of $\mathrm{AD}$, is still an expensive procedure. Amyloid PET imaging, which provides pathophysiological information such as the accumulation of $A \beta$ plaques, besides being expensive, is still not available in all dementia centers. CSF analysis, which provides pathophysiological information on both tau and $A \beta$ accumulation, is an invasive procedure with an albeit low but possible risk of complications and cannot be performed in patients on anticoagulation therapy. In this study, we have shown that TMS assessment added to the clinical and neuropsychological evaluation may increase diagnostic accuracy up to $98 \%$, comparable to that obtained from the addition of amyloidosis biomarkers, which reached 99\% accuracy.

Concurrently, in the aging population, the prevalence of $\mathrm{AD}$ and other neurodegenerative dementias is steadily increasing worldwide [1], thus making it crucial to find accurate but inexpensive markers that can be used to screen at-risk populations. Furthermore, ideal markers should be easy to measure and noninvasive to be implemented in secondary referral centers.

In this work, we confirmed previous literature data suggesting that the use of biomarkers increased DCAD in clinical settings as compared with clinical and neuropsychological evaluation alone [12], and we proposed

Table 2 Performance of the three different assessment sections in discriminating between Alzheimer disease and frontotemporal dementia in terms of diagnostic confidence of Alzheimer disease

\begin{tabular}{|c|c|c|c|c|c|c|}
\hline \multirow{2}{*}{$\begin{array}{l}\text { Indices of separation } \\
\text { Cluster mean }\end{array}$} & \multicolumn{2}{|l|}{ Clinical work-up } & \multicolumn{2}{|c|}{ Clinical work-up plus amyloid markers } & \multicolumn{2}{|c|}{ Clinical work-up plus TMS markers } \\
\hline & Mean $(95 \% \mathrm{Cl})$ & $p$ Value & Mean $(95 \% \mathrm{Cl})$ & $p$ Value & Mean $(95 \% \mathrm{Cl})$ & $p$ Value \\
\hline AD cluster & $75.0(73.4-76.6)$ & $p<1.0 \times 10^{-5}$ & $87.1(85.2-89.0)$ & $p<1.0 \times 10^{-7}$ & $89.3(87.9-90.7)$ & $p<1.0 \times 10^{-10}$ \\
\hline FTD cluster & $19.9(18.2-21.6)$ & & $11.1(9.0-13.2)$ & & $9.3(7.6-10.9)$ & \\
\hline AD vs FTD cluster centroid distance & 55.1 & & 76.0 & & 80.0 & \\
\hline
\end{tabular}

Abbreviations: AD Alzheimer disease, FTD Frontotemporal dementia, TMS Transcranial magnetic stimulation

DCAD mean (and $95 \% \mathrm{Cl}$ ) of the two clusters correspond to the means of the two estimated Gaussian components of each mixture model. P-value refers to the difference between cluster means within each assessment 
Table 3 Association (measured by OR) and performance (measured by AUC) evaluation of diagnostic confidence of Alzheimer disease of each assessment section in predicting the "gold standard" diagnosis

\begin{tabular}{llll}
\hline Assessment & AUC (95\% Cl) & OR (95\% Cl) & $p$ Value \\
\hline Clinical work-up & $0.82(0.74-0.90)$ & $1.05(1.03-1.07)$ & $4.9 \times 10^{-9}$ \\
Clinical work-up plus amyloid markers & $0.99(0.98-1)$ & $1.11(1.08-1.16)$ & $6.1 \times 10^{-8}$ \\
Clinical work-up plus TMS & $0.98(0.96-1)$ & $1.32(1.11-1.83)$ & 0.046
\end{tabular}

TMS Transcranial magnetic stimulation

$p$ Value refers to the statistical significance of ORs

AUC 95\% Cl: If Cl includes 0.5, the AUC is statistically not different from an AUC obtained from a classification by chance

TMS intracortical connectivity measures as a noninvasive diagnostic tool to be added to the clinical work-up.

TMS intracortical connectivity measures may evaluate the other side of the coin of AD pathophysiology: Instead of targeting amyloid deposition by measuring CSF $\mathrm{A} \beta_{1-42}$ or binding of amyloid brain imaging ligands on PET, TMS measures assess neurotransmitter deficits, namely the well-established impairment in cholinergic transmission observed in AD [27, 57].

Another well-established hallmark of AD is the impairment in synaptic plasticity observed in animal models of disease [58]. Concurrently, in vivo correlates of long-term potentiation (LTP)-like plasticity, evaluated with paired associative stimulation (PAS) protocols [59] or repetitive TMS $[57,60]$, have shown a significant impairment of LTP-like cortical plasticity in patients with AD. These studies have been limited to the evaluation of the motor cortex, however, which is probably not the most affected brain region in AD. This restriction has been exceeded by using TMS-electroencephalogram coregistration techniques in brain regions other than the motor cortex, such as the dorsolateral prefrontal cortex [61] or precuneus [62], confirming previous findings. However, in FTD, few studies have also shown a significant impairment in LTP-like plasticity evaluated with PAS in both genetic and sporadic FTD [18, 63]. It is still debated if plasticity protocols might aid in the discrimination between FTD and AD, and specific studies are currently lacking.

Some limitations of the present study need to be acknowledged. First, this is a monocentric study conducted in a tertiary referral center, and we evaluated the differential diagnosis of only two neurodegenerative disorders. Multicenter studies, including secondary referral centers and considering a broader spectrum of neurodegenerative dementias, are needed. Moreover, we conducted a retrospective study using medical records; thus, the evaluation of the add-on TMS parameters' value in DCAD should be addressed further in real-world situations.

\section{Conclusions}

Our data suggest that TMS measures increase discrimination performance when added to the clinical and neuropsychological evaluation with levels comparable to those of established biomarkers of brain amyloidosis. If these results were corroborated in larger samples, including subjects with mild cognitive impairment, TMS intracortical connectivity measures hold promise to be considered a helpful screening marker to be added to currently available diagnostic tools. Further studies considering both accuracy and economic burden of potential biomarkers are warranted.

\section{Additional file}

Additional file 1: Figure S1. ROC curves for clinical work-up, clinical work-up plus amyloid markers, and clinical work-up plus TMS. TMS Transcranial magnetic stimulation. (TIF $428 \mathrm{~kb}$ )

\section{Acknowledgements}

This study was supported by grants from "AIRAlzh Onlus" and "ANCC-COOP" issued (to VC).

Availability of data and materials

Owing to ethical restrictions protecting patient privacy, data are available from the corresponding author upon reasonable request.

Authors' contributions

$A B, A A, C F$, and $B B$ conceived of and designed the study. $A B, A A, C F, V C, V D$, $R T, M S C, G B, B P, G K, A P$, and $B B$ acquired and analyzed data. $A B, C F$, and $B B$ drafted the manuscript and figures. All coauthors revised the manuscript for important intellectual content. All authors read and approved the final manuscript.

Ethics approval and consent to participate

The study protocol was approved by the local ethics committee (NP1965; approved 19 May 2015).

Consent for publication

Not applicable.

Competing interests

The authors declare that they have no competing interests.

\section{Publisher's Note}

Springer Nature remains neutral with regard to jurisdictional claims in published maps and institutional affiliations.

\section{Author details}

${ }^{1}$ Neurology Unit, Department of Clinical and Experimental Sciences, University of Brescia, Brescia, Italy. ${ }^{2}$ IRCCS Centro San Giovanni di Dio Fatebenefratelli, Brescia, Italy. ${ }^{3}$ Department of Neuroscience, Psychology, Drug Research and Child Health, University of Florence, Florence, Italy. ${ }^{4}$ Neurology Unit, Ospedale Vallecamonica, Esine, Brescia, Italy. ${ }^{5}$ Nuclear Medicine Unit, Spedali Civili Brescia, Brescia, Italy. ${ }^{6}$ Non Invasive Brain 
Stimulation Unit, IRCCS Santa Lucia Foundation, Rome, Italy. ${ }^{7}$ Stroke Unit, Policlinico Tor Vergata, Rome, Italy.

\section{Received: 14 May 2018 Accepted: 28 August 2018 Published online: 18 September 2018}

\section{References}

1. Prince M, Bryce R, Albanese E, Wimo A, Ribeiro W, Ferri CP. The global prevalence of dementia: a systematic review and metaanalysis. Alzheimers Dement. 2013:9:63-75 e2.

2. Elahi FM, Miller BL. A clinicopathological approach to the diagnosis of dementia. Nat Rev Neurol. 2017;13:457-76.

3. Frisoni GB, Boccardi M, Barkhof F, Blennow K, Cappa S, Chiotis K, et al. Strategic roadmap for an early diagnosis of Alzheimer's disease based on biomarkers. Lancet Neurol. 2017;16:661-76.

4. Dubois B, Feldman HH, Jacova C, Hampel H, Molinuevo JL, Blennow K, et al. Advancing research diagnostic criteria for Alzheimer's disease: the IWG-2 criteria. Lancet Neurol. 2014:13:614-29.

5. McKhann GM, Knopman DS, Chertkow H, Hyman BT, Jack CR, Kawas CH, et al. The diagnosis of dementia due to Alzheimer's disease: recommendations from the National Institute on Aging-Alzheimer's association workgroups on diagnostic guidelines for Alzheimer's disease. Alzheimers Dement. 2011;7: 263-9.

6. Blennow $\mathrm{K}$, Hampel $\mathrm{H}$, Zetterberg $\mathrm{H}$. Biomarkers in amyloid- $\beta$ immunotherapy trials in Alzheimer's disease. Neuropsychopharmacology. 2014:39:189-201

7. Andrieu S, Coley N, Lovestone S, Aisen PS, Vellas B. Prevention of sporadic Alzheimer's disease: lessons learned from clinical trials and future directions. Lancet Neurol. 2015;14:926-44.

8. Shaw LM, Vanderstichele H, Knapik-Czajka M, Clark CM, Aisen PS, Petersen $\mathrm{RC}$, et al. Cerebrospinal fluid biomarker signature in Alzheimer's Disease Neuroimaging Initiative subjects. Ann Neurol. 2009;65:403-13.

9. Clark CM, Pontecorvo MJ, Beach TG, Bedell BJ, Coleman RE, Doraiswamy PM, et al. Cerebral PET with florbetapir compared with neuropathology at autopsy for detection of neuritic amyloid- $\beta$ plaques: a prospective cohort study. Lancet Neurol. 2012;11:669-78.

10. de Souza LC, Lamari F, Belliard S, Jardel C, Houillier C, De Paz R, et al. Cerebrospinal fluid biomarkers in the differential diagnosis of Alzheimer's disease from other cortical dementias. J Neurol Neurosurg Psychiatry. 2011; $82: 240-6$.

11. Ikonomovic MD, Klunk WE, Abrahamson EE, Mathis CA, Price JC, Tsopelas $\mathrm{ND}$, et al. Post-mortem correlates of in vivo PiB-PET amyloid imaging in a typical case of Alzheimer's disease. Brain. 2008;131:1630-45.

12. Boccardi M, Altomare D, Ferrari C, Festari C, Guerra UP, Paghera B, et al. Assessment of the incremental diagnostic value of florbetapir $F 18$ imaging in patients with cognitive impairment: the Incremental Diagnostic Value of Amyloid PET with $\left[{ }^{18} \mathrm{~F}\right]$-Florbetapir (INDIA-FBP) Study. JAMA Neurol. 2016;73: 1417-24.

13. Visser PJ, Verhey F, Knol DL, Scheltens P, Wahlund LO, Freund-Levi Y, et al. Prevalence and prognostic value of CSF markers of Alzheimer's disease pathology in patients with subjective cognitive impairment or mild cognitive impairment in the DESCRIPA study: a prospective cohort study. Lancet Neurol. 2009;8:619-27.

14. Borroni B, Premi E, Di Luca M, Padovani A. Combined biomarkers for early Alzheimer disease diagnosis. Curr Med Chem. 2007;14:1171-8.

15. Benussi A, Di Lorenzo F, Dell'Era V, Cosseddu M, Alberici A, Caratozzolo S, et al. Transcranial magnetic stimulation distinguishes Alzheimer disease from frontotemporal dementia. Neurology. 2017:89:665-72.

16. Padovani A, Benussi A, Cantoni V, Dell'Era V, Cotelli MS, Caratozzolo S, et al. Diagnosis of mild cognitive impairment due to Alzheimer's disease with transcranial magnetic stimulation. J Alzheimers Dis. 2018;65:221-30.

17. Benussi A, Dell'Era V, Cantoni V, Ferrari C, Caratozzolo S, Rozzini L, et al. Discrimination of atypical parkinsonisms with transcranial magnetic stimulation. Brain Stimul. 2018;11:366-73.

18. Benussi A, Cosseddu M, Filareto I, Dell'Era V, Archetti S, Sofia Cotelli M, et al. Impaired long-term potentiation-like cortical plasticity in presymptomatic genetic frontotemporal dementia. Ann Neurol. 2016:80:472-6.

19. Francis PT, Palmer AM, Snape M, Wilcock GK. The cholinergic hypothesis of Alzheimer's disease: a review of progress. J Neurol Neurosurg Psychiatry. 1999;66:137-47.
20. Freitas C, Mondragón-Llorca H, Pascual-Leone A. Noninvasive brain stimulation in Alzheimer's disease: systematic review and perspectives for the future. Exp Gerontol. 2011;46:611-27.

21. Teipel SJ, Meindl T, Grinberg L, Grothe M, Cantero JL, Reiser MF, et al. The cholinergic system in mild cognitive impairment and Alzheimer's disease: an in vivo MRI and DTI study. Hum Brain Mapp. 2011;32:1349-62.

22. Mufson EJ, Binder L, Counts SE, Dekosky ST, de Toledo-Morrell L, Ginsberg SD, et al. Mild cognitive impairment: pathology and mechanisms. Acta Neuropathol. 2012;123:13-30.

23. Levenga J, Krishnamurthy $\mathrm{P}$, Rajamohamedsait $\mathrm{H}$, Wong $\mathrm{H}$, Franke TF, Cain $P$, et al. Tau pathology induces loss of GABAergic interneurons leading to altered synaptic plasticity and behavioral impairments. Acta Neuropathol Commun. 2013;1:34

24. Majumder P, Chen YT, Bose JK, Wu CC, Cheng WC, Cheng SJ, et al. TDP-43 regulates the mammalian spinogenesis through translational repression of Rac1. Acta Neuropathol. 2012;124:231-45.

25. McDonnell MN, Orekhov $Y$, Ziemann $U$. The role of GABAB receptors in intracortical inhibition in the human motor cortex. Exp Brain Res. 2006;173:86-93.

26. Murley AG, Rowe JB. Neurotransmitter deficits from frontotemporal lobar degeneration. Brain. 2018:141:1263-85.

27. Di Lazzaro V, Oliviero A, Tonali PA, Marra C, Daniele A, Profice P, et al. Noninvasive in vivo assessment of cholinergic cortical circuits in AD using transcranial magnetic stimulation. Neurology. 2002;59:392-7.

28. Tan RH, Kril JJ, Yang Y, Tom N, Hodges JR, Villemagne VL, et al. Assessment of amyloid $\beta$ in pathologically confirmed frontotemporal dementia syndromes. Alzheimers Dement (Amst). 2017;9:10-20.

29. Procter AW, Qurne M, Francis PT. Neurochemical features of frontotemporal dementia. Dement Geriatr Cogn Disord. 1999;10:80-4.

30. Hardy J, Cowburn R, Barton A, Reynolds G, Dodd P, Wester P, et al. A disorder of cortical GABAergic innervation in Alzheimer's disease. Neurosci Lett. 1987:73:192-6.

31. Dermaut B, Kumar-Singh S, Rademakers R, Theuns J, Cruts M, Van Broeckhoven C. Tau is central in the genetic Alzheimer-frontotemporal dementia spectrum. Trends Genet. 2005;21:664-72.

32. Gorno-Tempini ML, Hillis AE, Weintraub S, Kertesz A, Mendez M, Cappa SF, et al. Classification of primary progressive aphasia and its variants. Neurology. 2011;76:1006-14.

33. Rascovsky K, Hodges JR, Knopman D, Mendez MF, Kramer JH, Neuhaus J, et al. Sensitivity of revised diagnostic criteria for the behavioural variant of frontotemporal dementia. Brain. 2011;134:2456-77.

34. Chare L, Hodges JR, Leyton CE, McGinley C, Tan RH, Kril JJ, et al. New criteria for frontotemporal dementia syndromes: clinical and pathological diagnostic implications. J Neurol Neurosurg Psychiatry. 2014:85:865-70

35. Perry DC, Brown JA, Possin KL, Datta S, Trujillo A, Radke A, et al. Clinicopathological correlations in behavioural variant frontotemporal dementia. Brain. 2017;140:3329-45.

36. Magni E, Binetti G, Bianchetti A, Rozzini R, Trabucchi M. Mini-Mental State Examination: a normative study in Italian elderly population. Eur J Neurol. 1996:3:198-202

37. Morris JC. The clinical dementia rating (CDR): current version and scoring rules. Neurology. 1993:43:2412.

38. Novelli G, Papagno C, Capitani E, Laiacona M, Vallar G, Cappa SF. Three clinical tests for the assessment of verbal long-term memory function: norms from 320 normal subjects [in Italian]. Arch Psicol Neurol Psichiatr. 1986:47:278-96.

39. Caffarra P, Vezzadini G, Dieci F, Zonato F, Venneri A. Rey-Osterrieth complex figure: normative values in an Italian population sample. Neurol Sci. 2002:22:443-7.

40. Giovagnoli AR, Del Pesce M, Mascheroni S, Simoncelli M, Laiacona M, Capitani E. Trail Making Test: normative values from 287 normal adult controls. Ital J Neurol Sci. 1996;17:305-9.

41. Novelli G, Papagno C, Capitani E, Laiacona M, Vallar G, Cappa SF. Three clinical tests to research and rate the lexical performance of normal subjects [in Italian]. Arch Psicol Neurol Psichiatr. 1986:47:477-506.

42. Katz S, Ford AB, Moskowitz RW, Jackson BA, Jaffe MW. Studies of illness in the aged. The index of ADL: a standardized measure of biological and psychosocial function. JAMA. 1963;185:914-9.

43. Lawton MP. Brody EM. Assessment of older people: self-maintaining and instrumental activities of daily living. Gerontologist. 1969;9:179-86. 
44. Cummings JL, Mega M, Gray K, Rosenberg-Thompson S, Carusi DA, Gornbein J. The Neuropsychiatric Inventory: comprehensive assessment of psychopathology in dementia. Neurology 1994;44:2308-8.

45. Borroni B, Benussi A, Archetti S, Galimberti D, Parnetti L, Nacmias B, et al. CSF p-Tau ${ }_{181} /$ Tau ratio as biomarker for TDP pathology in frontotemporal dementia. Amyotroph Lateral Scler Frontotemporal Degener. 2015;16:86-91.

46. Benussi A, Cotelli MS, Cosseddu M, Bertasi V, Turla M, Salsano E, et al. Preliminary results on long-term potentiation-like cortical plasticity and cholinergic dysfunction after miglustat treatment in Niemann-Pick disease type C. JIMD Rep. 2017;36:19-27.

47. Rossini PM, Burke D, Chen R, Cohen LG, Daskalakis Z, Di lorio R, et al. Noninvasive electrical and magnetic stimulation of the brain, spinal cord, roots and peripheral nerves: basic principles and procedures for routine clinical and research application. An updated report from an I.F.C.N. Committee. Clin Neurophysiol. 2015:126:1071-107.

48. Kujirai T, Caramia MD, Rothwell JC, Day BL, Thompson PD, Ferbert A, et al. Corticocortical inhibition in human motor cortex. J Physiol. 1993;471:501-19.

49. Ziemann U, Rothwell JC, Ridding MC. Interaction between intracortical inhibition and facilitation in human motor cortex. J Physiol. 1996;496:873-81.

50. Tokimura H, Di Lazzaro V, Tokimura Y, Oliviero A, Profice P, Insola A, et al. Short latency inhibition of human hand motor cortex by somatosensory input from the hand. J Physiol. 2000;523:503-13 A published erratum appears in J Physiol. 2000;524:942.

51. McLachlan G, Peel D. Finite mixture models. Hoboken, NJ: Wiley; 2005.

52. Fraley C, Raftery A. Model-based methods of classification: using the mclust software in chemometrics. J Stat Softw. 2007:18(6):1-13.

53. Dempster AP, Laird NM, Rubin DB. Maximum likelihood from incomplete data via the EM algorithm. J R Stat Soc. 1977;39:1-38.

54. Burnham KP, Anderson DR, Huyvaert KP. AIC model selection and multimodel inference in behavioral ecology: some background, observations, and comparisons. Behav Ecol Sociobiol. 2011;65:23-35.

55. Rabinovici GD, Rosen HJ, Alkalay A, Kornak J, Furst AJ, Agarwal N, et al. Amyloid vs FDG-PET in the differential diagnosis of AD and FTLD. Neurology. 2011;77:2034-42.

56. Irwin DJ, Trojanowski JQ, Grossman M. Cerebrospinal fluid biomarkers for differentiation of frontotemporal lobar degeneration from Alzheimer's disease. Front Aging Neurosci. 2013;5:6.

57. Di Lorenzo F, Ponzo V, Bonnì S, Motta C, Negrão Serra PC, Bozzali M, et al. Long-term potentiation-like cortical plasticity is disrupted in Alzheimer's disease patients independently from age of onset. Ann Neurol. 2016;80:202-10.

58. Palop JJ, Mucke L. Amyloid- $\beta$-induced neuronal dysfunction in Alzheimer's disease: from synapses toward neural networks. Nat Neurosci. 2010;13:812-8

59. Battaglia F, Wang HY, Ghilardi MF, Gashi E, Quartarone A, Friedman E, et al. Cortical plasticity in Alzheimer's disease in humans and rodents. Biol Psychiatry. 2007:62:1405-12.

60. Koch G, Di Lorenzo F, Bonnì S, Ponzo V, Caltagirone C, Martorana A. Impaired LTP- but not LTD-like cortical plasticity in Alzheimer's disease patients. J Alzheimers Dis. 2012;31:593-9.

61. Kumar S, Zomorrodi R, Ghazala Z, Goodman MS, Blumberger DM, Cheam A, et al. Extent of dorsolateral prefrontal cortex plasticity and its association with working memory in patients with Alzheimer disease. JAMA Psychiatry. 2017;74:1266-74

62. Koch G, Bonnì S, Pellicciari MC, Casula EP, Mancini M, Esposito R, et al. Transcranial magnetic stimulation of the precuneus enhances memory and neural activity in prodromal Alzheimer's disease. Neuroimage. 2018;169:302-11.

63. Borroni B, Benussi A, Premi E, Alberici A, Marcello E, Gardoni F, et al. Biological, neuroimaging, and neurophysiological markers in frontotemporal dementia: three faces of the same coin. J Alzheimers Dis. 2018:62:1113-23.

Ready to submit your research? Choose BMC and benefit from:

- fast, convenient online submission

- thorough peer review by experienced researchers in your field

- rapid publication on acceptance

- support for research data, including large and complex data types

- gold Open Access which fosters wider collaboration and increased citations

- maximum visibility for your research: over $100 \mathrm{M}$ website views per year

At BMC, research is always in progress.

Learn more biomedcentral.com/submissions 